\title{
Hipocalcemia severa por deficiencia de vitamina $D$ en una adulta mayor
}

\author{
Deficiency of vitamin D as cause of severe hypocalcemia in an old woman \\ Otto Barnaby Guillén López 1,a;2,b

\section{RESUMEN} \\ La deficiencia de vitamina $\mathrm{D}$ es una condición de muy alta prevalencia en adultos mayores y puede presentarse \\ como hipocalcemia sintomática. Se presenta un caso de una mujer de 75 años con convulsiones generalizadas por \\ hipocalcemia con función renal y paratiroidea normales en la que se demostró niveles deficientes de vitamina D.
}

PALABRAS CLAVE: Deficiencia de vitamina D, hipocalcemia, ancianos. (Fuente: DeCS BIREME).

\section{SUMMARY}

Vitamin D deficiency is a very high prevalence condition in older adults and may present as symptomatic hypocalcemia. I present a case of a 75-year-old woman with generalized convulsions due to hypocalcaemia with normal renal and parathyroid function in which vitamin D levels were deficient.

KEYWORDS: Vitamin D deficiency, hypocalcemia. aged. (Source: MeSH NLM).

\section{INTRODUCCIÓN}

La deficiencia de vitamina $\mathrm{D}$ es una enfermedad prevalente, sobretodo en la población adulta mayor, que tiene numerosas consecuencias en el sistema musculoesquelético (osteomalacia, predisposición a fracturas, dolores y debilidad muscular proximales, entre otros síntomas) (1); sin embargo, es subdiagnosticada y, por lo tanto, no es tratada (2).

Niveles de 25 hidroxi-vitamina D (25OHD) de más de $24 \mathrm{ng} / \mathrm{ml}$ se asocian con menores riesgos de fractura de cadera, no vertebrales y de caídas, y con una adecuada función neuromuscular (3), por ello, la mayoría de los reportes de prevalencia de déficit de vitamina $\mathrm{D}$ diferencian dos términos: deficiencia (25OHD de $20 \mathrm{ng} / \mathrm{ml}$ o menos) e insuficiencia (25OHD menos de $30 \mathrm{ng} / \mathrm{ml}$ ) de vitamina $\mathrm{D}$.
Se han encontrado elevadas prevalencias de deficiencia de vitamina $\mathrm{D}$ en adultos mayores en asilos (25-50\%), en consulta ambulatoria (44\%), con osteoporosis $(30 \%)$, con fractura de cadera $(23 \%)$ y hospitalizados (57\%) (2); sin embargo, en Sudamérica existen pocos reportes de prevalencia de deficiencia de vitamina D en la población adulta mayor. González (4), realizó una revisión sistemática de los datos publicados en Argentina, Brasil y Chile, encontrando que entre $47 \%$ y $77 \%$ de adultos mayores presentan niveles disminuidos de vitamina $\mathrm{D}$ en los meses de invierno comparado con un menor porcentaje (15$57 \%$ ) en los meses de verano.

En el Perú, solo existe una investigación que reporta que $87,5 \%$ mujeres postmenopáusicas con osteoporosis primaria tuvo deficiencia de vitamina $\mathrm{D}$ (5). Por ello, el motivo del reporte fue mostrar un caso

\footnotetext{
Hospital Nacional Arzobispo Loayza. Lima, Perú.

Facultad de Medicina, Universidad Peruana Cayetano Heredia. Lima, Perú.

Médico Internista;

Profesor Auxiliar.
} 
de deficiencia de vitamina $\mathrm{D}$ con consecuencia grave en una mujer adulta mayor, debido a que a pesar de ser de alta prevalencia, no existen reportes sobre el tema en el Perú desde el 2011.

\section{PRESENTACIÓN DEL CASO}

Mujer de raza mestiza de 75 años de edad admitida en el servicio de emergencia del Hospital Arzobispo Loayza (Lima, Perú) por presentar 15 minutos antes convulsiones generalizadas tónico clónicas con relajación de esfínteres y pérdida de conciencia por 5 minutos. No presentaba cefalea o fiebre previas.

Tenía el antecedente de hipertensión arterial de 22 años de evolución en tratamiento con Losartán; fibrilación auricular e insuficiencia cardíaca desde hacía tres años en tratamiento con furosemida $40 \mathrm{mg}$ por día e infarto agudo de miocardio de hace dos años en tratamiento con aspirina y atorvastatina. No había historia de diabetes mellitus, enfermedad tiroidea, enfermedad cerebrovascular ni de intervenciones quirúrgicas previas.

$\mathrm{Al}$ examen físico de ingreso su presión arterial (PA) fue 180/90 mm Hg, frecuencia cardíaca (FC) 64 latidos por minuto, FR en 24 respiraciones por minuto y saturación de oxígeno $91 \%$ con oxígeno ambiental.
En el examen neurológico presentó somnolencia, estaba orientada en persona pero desorientada en tiempo y espacio, escala de Glasgow de 13 y hemiparesia izquierda y signo de Babinsky izquierdo. No había signos meníngeos. No se examinó por signos de Trosseau o Chvostek. No tuvo traumatismos o fracturas secundarias a la convulsión.

Los exámenes de laboratorio de ingreso mostraron calcio iónico $0,54 \mathrm{mmol} / \mathrm{l}$ (VN: 1,16-1,32); paratohormona (PTH) en $36 \mathrm{pg} / \mathrm{dl}$ (VN: 15-65); glicemia $152 \mathrm{mg} / \mathrm{dl}$; creatinina $1,0 \mathrm{mg} / \mathrm{dl}$; sodio: 133 $\mathrm{mEq} / \mathrm{l}$; potasio $4,2 \mathrm{mEq} / \mathrm{l}$; y hemoglobina glicosilada $8,1 \%$; hematocrito, leucocitos, plaquetas, y pruebas de coagulación, normales. No se determinó fósforo, magnesio ni $\mathrm{pH}$ séricos ni calcio urinario. En la tomografía cerebral sin contraste no se evidenció tumoraciones, hemorragia ni isquemia aguda; sólo mostró atrofia cerebral leve difusa sin hidrocefalia.

En emergencia recibió tratamiento con fenitoína $1500 \mathrm{mg}$ en infusión endovenosa (EV) y al siguiente día fue hospitalizada en el servicio de medicina sin presentar otra convulsión y con estado de conciencia recuperado. Recibió infusión de $50 \mathrm{ml}$ de gluconato de calcio al 10\% diluidos en $950 \mathrm{ml}$ de dextrosa al 5\% por vía EV en 24 horas, y luego carbonato de calcio 1500 mg VO por día. Además recibió bisoprolol,

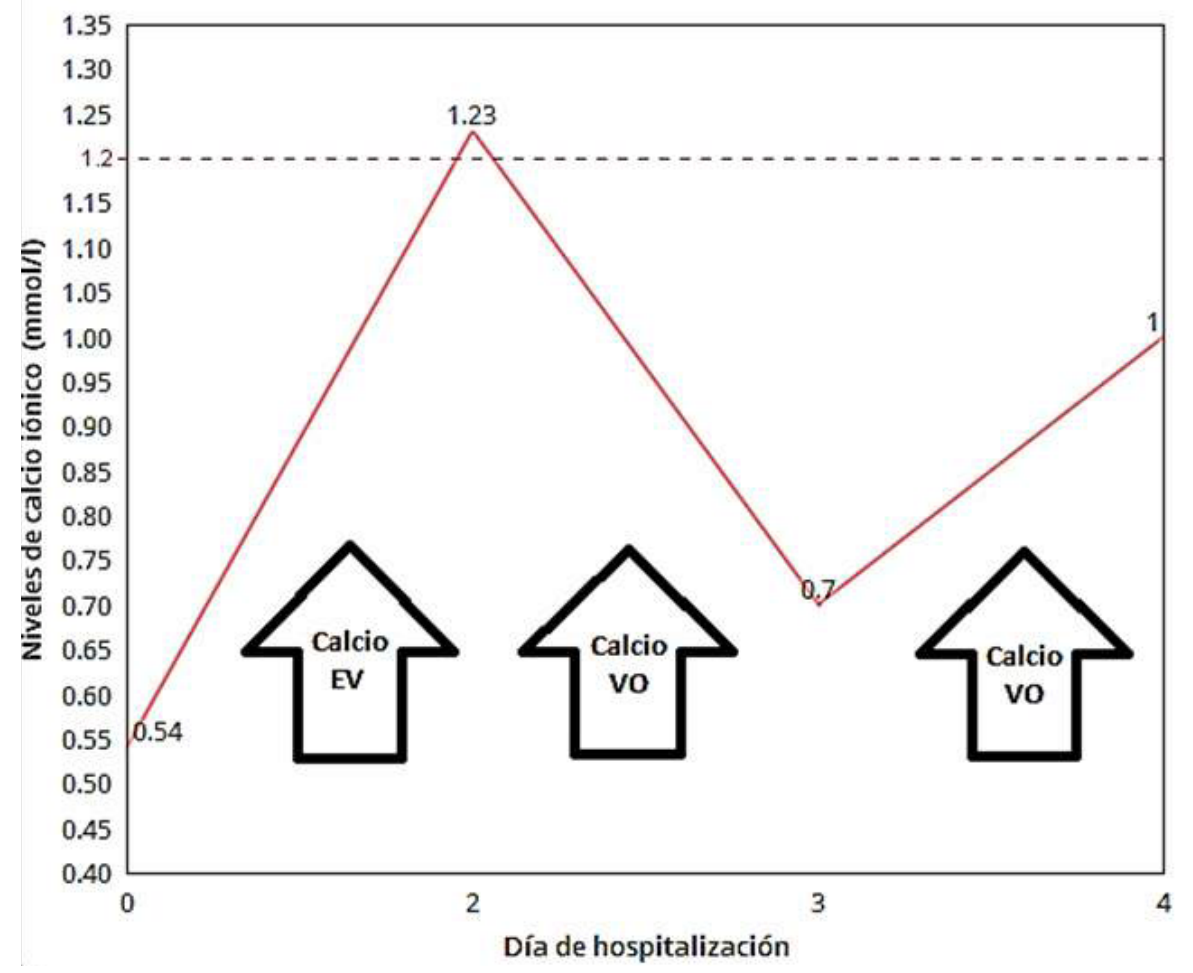

Figura 1. Niveles de calcio iónico durante su hospitalización. 
metformina, aspirina y atorvastatina. No reinició la terapia con furosemida ni recibió fenitoína de mantenimiento.

A pesar que la paciente no presentó convulsiones y había recuperado totalmente el estado de conciencia, no se tenía explicación de la hipocalcemia, por lo que se le solicitó dosaje de vitamina D (25 OHD) sérica cuyo resultado fue $11 \mathrm{ng} / \mathrm{ml}$ (VN: 30-100), lo que motivó el inicio de terapia de reemplazo de vitamina $\mathrm{D}$ vía oral. La evolución de los valores de calcio iónico se observa en la figura 1.

La paciente salió de alta al noveno día de hospitalización recuperada, con solo leve hemiparesia izquierda y con glicemia, FC y PA controladas.

\section{DISCUSIÓN}

El caso reportado describe a una paciente adulta mayor que se presentó súbitamente con convulsiones tardías sin antecedente de trauma craneal, sin clínica de meningitis, y sin demostrarse alteraciones orgánicas cerebrales ni del sodio o glucosa, las cuales son algunas de las causas más frecuentes de convulsiones tardías en adultos mayores (6). Solo se demostró hipocalcemia severa, la cual puede ser causa de convulsiones en cualquier grupo etario. Aunque llama la atención que no se haya evidenciado signos de tetania, se describe que hasta en $29 \%$ de pacientes con hipocalcemia severa puede estar ausente el signo de Chvostek (7). Igual la hipocalcemia severa se corrige en emergencia con reposición de calcio endovenoso y luego vía oral, pero siempre se debe buscar la etiología para tratarla y, de ser factible, evitar la reaparición de la hipocalcemia.

Siguiendo el algoritmo propuesto por Fukumoto et al (8), que, para un paciente con hipocalcemia sin insuficiencia renal o hipoparatiroidismo, debe investigarse una probable deficiencia de vitamina $\mathrm{D}$, la paciente reportada tuvo función renal con niveles de PTH normales y se demostró la deficiencia de vitamina $\mathrm{D}$ en valores menores de $20 \mathrm{ng} / \mathrm{ml}$ que podría haber sido una de las causas de la hipocalcemia severa. Cooper y Gittoes, describen que se deben investigar los valores de magnesio y de fosfatasa alcalina séricos para definir la etiología de hipocalcemia, ellos mencionan que la hipomagnesemia, es una causa rara de hipocalcemia (7). Otras enfermedades raras causantes de hipocalcemia que pueden descartarse en esta paciente son la resistencia a PTH o metástasis óseas escleróticas, pues dichas patologías cursan con PTH elevada.
Se ha reportado un caso semejante de primera convulsión por deficiencia de vitamina $\mathrm{D}$ en Turquía (9) en una mujer de 63 años con osteoporosis que vivía sola, pasaba todo el día en casa sin exposición adecuada al sol y tenía ingesta baja de pescado y leche (9). En nuestra paciente no se indagó sobre estas características.

Generalmente se debe obtener alrededor de 2000 UI de vitamina D con una exposición al sol durante 20 a 30 minutos en cara y antebrazos en horas del mediodía (1), pero esto normalmente no se puede realizar en los meses de invierno. Sin embargo, a pesar de tener la fuente solar de rayos ultravioleta B durante el resto del año, la exposición al sol es cada vez más infrecuente por distintas razones: trabajar en lugares cerrados durante las horas del día, pasar la mayor parte del día sin salir de casa (por ejemplo, adultos mayores o personas postradas o con alguna discapacidad que impida movilizarse), vivir en el interior de edificios grandes donde no hay luz solar, usar protectores solares (sobre todo en el verano), usar ropa que cubra los antebrazos y piernas (por ejemplo, adultos mayores con desregulación de la temperatura corporal), usar sombreros o gorras, entre otros.

Por otro lado, se piensa que vivir en latitudes cercanas al ecuador como el Perú, favorece una adecuada exposición al sol para mantener niveles adecuados de vitamina $\mathrm{D}$ en la población. Sin embargo, Orces encontró en Ecuador que 68\% de adultos mayores tenían niveles de vitamina $\mathrm{D}$ menos de $30 \mathrm{ng} / \mathrm{ml}$, con niveles más bajos en mujeres, que viven solas, y en áreas montañosas en los Andes, que consumen productos lácteos menos de una vez al día, que hacen actividad física menos de 3 veces por semana, y que tienen discapacidad para movilizarse (10). Estas características son similares en nuestra población, ya que solo $11,6 \%$ de adultos mayores de 60 años realiza actividad física diaria o interdiaria (11), y, por otro lado, el Perú ocupa el penúltimo lugar en Latinoamérica en el consumo de leche y derivados (5).

Una ingesta adecuada de vitamina D es de 400 a 600 UI por día, y aunque la vitamina D se obtiene de alimentos como la yema de huevo, pescados grasos, hígado y leche enriquecida, el consumo de vitamina D es bajo en Estados Unidos y en países de Europa (1). En Sudamérica, Brito et al., en su revisión sistemática encontraron que el promedio de ingesta de vitamina $\mathrm{D}$ en Brasil es mucho menor que la recomendada y oscila entre 60,7 y 124 UI por día, tanto en niños y 
adolescentes como en adultos y adultos mayores (12). Por su parte, en Chile se reporta que solo el $30 \%$ de la vitamina $\mathrm{D}$ puede ser obtenida por dieta (2).

Entonces son varias las razones que explicarían la alta prevalencia reportada de deficiencia de vitamina $\mathrm{D}$ en diferentes grupos etarios, pero sobre todo en la población adulta mayor, que reúnen varias razones para tener niveles séricos disminuidos de vitamina D. Incluso, se ha propuesto que la deficiencia de vitamina $\mathrm{D}$ puede ser un problema de salud pública en América Latina, no solo por su alta prevalencia sino por sus consecuencias no solo relacionadas al sistema osteomuscular, sino que se ha asociado también a riesgo incrementado de hipertiroidismo, resistencia a la insulina y mortalidad global y cardiovascular elevadas (11).

Podemos concluir que la deficiencia de vitamina $\mathrm{D}$ debe investigarse en adultos mayores, que además cursen con hipocalcemia sin afectación de la función renal y paratiroidea, no solo porque tiene consecuencias moderadas a graves en el sistema osteomuscular y endocrino, sino también mayor mortalidad.

Es necesario por ello realizar estudios de prevalencia de deficiencia de vitamina $\mathrm{D}$ en la población susceptible en el Perú, sea asintomática o con síntomas osteomusculares de cualquier grupo etario, ya que es muy probable que no se esté diagnosticando en muchos pacientes. Personas con osteoporosis, que trabajan en lugares cerrados durante el día (oficinistas, personal de salud, amas de casa, entre otros), obesos, desnutridos, con piel oscura, con problemas en la piel que usen bloqueadores solares diariamente, postradas o institucionalizadas, con insuficiencia renal crónica, adultos mayores y con síndromes de malabsorción (intestino corto, pancreatitis, cirugía bariátrica, etc.) deben ser investigados por posible deficiencia de vitamina D.

\section{Declaración de financiamiento y de conflictos de intereses:}

El trabajo fue financiado por el autor. Declara no tener algún conflicto de intereses.

\section{Correspondencia:}

Guillén López, Otto Barnaby

Correo electrónico: otto.guillen.1@upch.pe

\section{REFERENCIAS BIBLIOGRÁFICAS}

1. Pearce SHS, Cheetham TD. Diagnosis and management of vitamin D deficiency. BMJ. 2010; 340: b5664.

2. Barberán M, Aguilera G, Brunet L, Maldonado F. Déficit de vitamina D: Revisión epidemiológica actual. Rev Hosp Clín Univ Chile. 2014; 25:127- 34.

3. Kennel KA, Drake MT, Hurley DL. Vitamin D deficiency in adults: When to test and how to treat. Mayo Clin Proc. 2010;85(8):752-758.

4. González G. Vitamin D status among healthy postmenopausal women in South America, DermatoEndocrinology. 2013; 5(1): 117-120. DOI: https:// doi.org/10.4161/derm.24807

5. Sotelo W, Calvo A. Niveles de vitamina D en mujeres posmenopáusicas con osteoporosis primaria. Rev Med Hered. 2011; 22:10-14.

6. Li X, Breteler MM, Bruyne MC, Meinardi H, Hauser WA, Hofman, A. Vascular Determinants of epilepsy: The Rotterdam Study. Epilepsia. 1997; 38: 12161220. doi: https://doi.org/10.1111/j.1528-1157.1997. tb01219.x

7. Cooper MS, Gittoes NJL. Diagnosis and management of hypocalcaemia. BMJ. 2008; 336(7656):12981302. doi: https://doi.org/10.1136/bmj.39582.589433. BE

8. Fukumoto S, Namba N, Ozono K, et al. Causes and differential diagnosis of hypocalcemia : Recommendation proposed by expert panel supported by Ministry of Health, Labour and Welfare, Japan. Endocrine Journal. 2008; 55 (5):787-794.

9. Arpaci D, Yilmaz U, Yaylaci S, Çölbay M, Tamer A. First seizure presentation in an elderly woman with primary vitamin D deficiency: a case report. Turkish Journal of Geriatrics. 2014; 17(2):200-204.

10. Orces C. Vitamin D Status among older adults residing in the Littoral and Andes Mountains in Ecuador. The Scientific World Journal. 2015; 2015: 1-8. doi: http://dx.doi.org/10.1155/2015/545297

11. Gonzales-Mechán M. Actividad física y programa de ejercicio en el adulto mayor: Necesidades no aplicadas en su verdadera dimensión. Rev Cuerpo Med HNAAA. 2016; 9 (2):140-141.

12. Brito A, Cori H, Olivares M, Mujica MF, Cediel G, López de Romaña D. Less than adequate vitamin $\mathrm{D}$ status and intake in Latin America and the Caribbean: A problem of unknown magnitude. Food Nutr Bull. 2013; 34(1):52-64. 